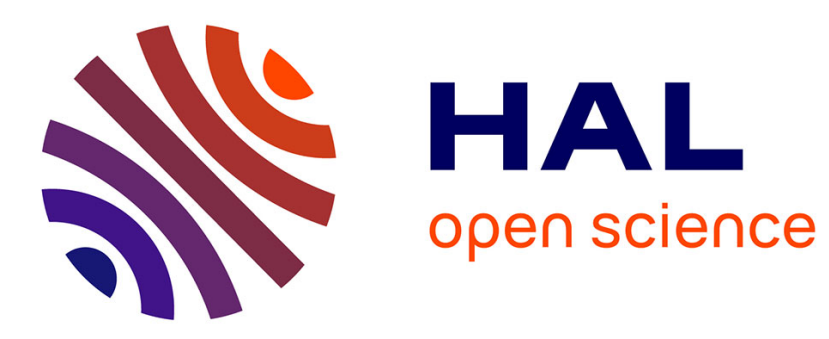

\title{
Kernel additive modeling for interference reduction in multi-channel music recordings
}

Thomas Prätzlich, Rachel Bittner, Antoine Liutkus, Meinard Müller

\section{To cite this version:}

Thomas Prätzlich, Rachel Bittner, Antoine Liutkus, Meinard Müller. Kernel additive modeling for interference reduction in multi-channel music recordings. IEEE International Conference on Acoustics, Speech and Signal Processing (ICASSP), Apr 2015, Brisbane, Australia. hal-01116686v2

\section{HAL Id: hal-01116686 \\ https://inria.hal.science/hal-01116686v2}

Submitted on 18 Feb 2015

HAL is a multi-disciplinary open access archive for the deposit and dissemination of scientific research documents, whether they are published or not. The documents may come from teaching and research institutions in France or abroad, or from public or private research centers.
L'archive ouverte pluridisciplinaire HAL, est destinée au dépôt et à la diffusion de documents scientifiques de niveau recherche, publiés ou non, émanant des établissements d'enseignement et de recherche français ou étrangers, des laboratoires publics ou privés. 


\title{
KERNEL ADDITIVE MODELING FOR INTERFERENCE REDUCTION IN MULTI-CHANNEL MUSIC RECORDINGS
}

\author{
Thomas Prätzlich ${ }^{1}$, Rachel M. Bittner ${ }^{2}$, Antoine Liutkus ${ }^{3}$, and Meinard Müller ${ }^{1}$ \\ ${ }^{1}$ International Audio Laboratories Erlangen, ${ }^{2}$ Music and Audio Research Lab, New York University, \\ ${ }^{3}$ Inria, speech processing team, Villers-lès-Nancy, France
}

\begin{abstract}
When recording a live musical performance, the different voices, such as the instrument groups or soloists of an orchestra, are typically recorded in the same room simultaneously, with at least one microphone assigned to each voice. However, it is difficult to acoustically shield the microphones. In practice, each one contains interference from every other voice. In this paper, we aim to reduce these interferences in multi-channel recordings to recover only the isolated voices. Following the recently proposed Kernel Additive Modeling framework, we present a method that iteratively estimates both the power spectral density of each voice and the corresponding strength in each microphone signal. With this information, we build an optimal Wiener filter, strongly reducing interferences. The trade-off between distortion and separation can be controlled by the user through the number of iterations of the algorithm. Furthermore, we present a computationally effective approximation of the iterative procedure. Listening tests demonstrate the effectiveness of the method.
\end{abstract}

Index Terms - audio source separation, multi-channel recordings, interference reduction, kernel additive modeling

\section{INTRODUCTION}

When recording musicians during a live performance, sound engineers typically set a microphone for each voice, which may be an instrument or an instrument group such as violin or brass sections in the case of an orchestra. Recordings are often made with the musicians playing together in the same room. See Figure 1 for an illustration. In a typical professional setup, the recording room is equipped with sound absorbing materials and acoustic shields to isolate all the voices as much as possible. However, complete acoustic isolation between the voices is often not possible. In practice and as depicted in Figure 1, each microphone not only records sound from its dedicated voice, but also from all others in the room, resulting in recordings that do not feature isolated signals, but rather mixtures of a predominant voice with all others being audible through what is referred to as interference, bleeding, crosstalk, or leakage. Such interferences are annoying in practice for several reasons. First, interferences greatly reduce the mixing possibilities for a sound engineer, and second, they prevent the removal or isolation of a voice from the recording, which may be desirable, e.g. for pedagogical reasons. A natural question thus arises: is it possible to remove these interferences to get clean, isolated voice signals?

This work has been supported by the BMBF project Freischütz Digital (Funding Code 01UG1239A to C). The International Audio Laboratories Erlangen are a joint institution of the Friedrich-Alexander-Universität Erlangen-Nürnberg (FAU) and Fraunhofer IIS.

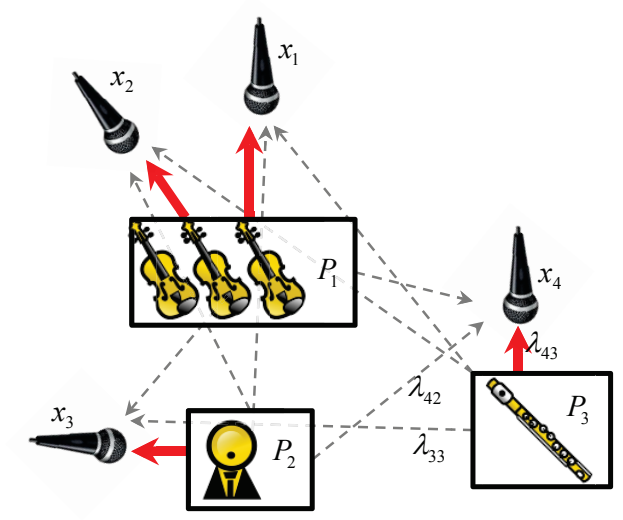

Fig. 1. Illustration of setup with three voices (violin section, flute, vocal soloist). Each voice is associated with at least one of the microphone channels. A solid line indicates a signal from a voice to its corresponding microphone, and a dashed line indicates an interference signal from other voices into a microphone.

In the past, several studies from the literature have considered this interference reduction problem. In each of these studies, it is assumed that for each voice there is at least one microphone present and that the number of voices and their corresponding microphones are known. While some approaches are based on echo cancellation and adaptive filtering involving estimation of propagation filters between voices and microphones in the time domain [1,2], others are based on Time-Frequency (TF) approaches, where the recordings are processed using adequate representation such as the Short Term Fourier Transform (STFT). For the latter cases, interference reduction is typically performed through Wiener filtering [3,4], which has been shown to produce very good results in terms of sound quality at a small computational cost. Interference reduction using Wiener filtering requires an estimate of the clean spectrogram for each voice. While [3] simply assumes that the spectrogram of each recording is already a good estimate for its dedicated voice signal, $[4,5]$ introduce further temporal constraints on the voices so as to better identify them from the mixture recordings. In [6], Non-Negative Matrix Factorization (NMF, see [7]) is used as a global parametric model for the voice spectrograms.

Interference Reduction (IR) is closely related to the problem of audio source separation, in which the objective is to separate a sound mixture into its constituent components [8]. Audio source separation in general is a very difficult problem where performance is highly dependent on the signals considered. However, recent studies demonstrate that separation methods can be very effective if prior 
information about the signals is available (see e.g. [9] and references therein).

In this paper, similar to [4], we exploit the fact that each voice is known to be predominant in its dedicated microphone channels. We also make use of a recently introduced framework for source separation called Kernel Additive Modeling (KAM, [10, 11, 12]) to exploit this strong prior information. The main contribution of this paper is the introduction of an algorithm based upon KAM which iteratively estimates the parameters of the model. We call this algorithm KAMIR: Kernel Additive Modeling for Interference Reduction. KAMIR is effectively a generalization of several previous methods; notably, [3] can be understood as a single iteration of KAMIR. Furthermore, we introduce an approximation that permits KAMIR to be computationally efficient while maintaining good performance.

The remainder of the paper is organized as follows: In Section 2 we describe the KAMIR model and the corresponding algorithm. In Section 3, we evaluate KAMIR through a perceptual evaluation test.

\section{MODEL AND METHOD}

\subsection{Notations and model}

Let $J$ be the number of voices and $I$ be the number of microphones. We will refer to the signal $x_{i}$ with $i \in\{1, \ldots, I\}$ from a microphone as a channel. In full generality, the sound produced by a particular voice $j \in\{1, \ldots, J\}$ can be heard in all channels $x_{i}$. For this reason, we define the image $y_{i j}$ as the contribution of the sound coming from voice $j$ in channel $x_{i}$. Channel $x_{i}$ is then simply taken as the sum of the corresponding voice images: $x_{i}=\sum_{j=1}^{J} y_{i j}$.

In the proposed technique, we first compute the Short-Term Fourier Transform (STFT) $X_{i}$ of each channel $x_{i}$. This yields $I$ matrices of dimension $N_{\omega} \times N_{t}$, where $N_{\omega}$ and $N_{t}$ are the number of frequency bands and the number of frames, respectively. Each point $(\omega, t)$ is called a Time-Frequency (TF) bin. We have:

$$
X_{i}(\omega, t)=\sum_{j=1}^{J} Y_{i j}(\omega, t)
$$

where $Y_{i j}$ denotes the $N_{\omega} \times N_{t}$ STFT of $y_{i j}$.

Following previous work in source separation literature (see e.g. [13]), we assume that all TF bins of the audio signals are independent. Furthermore, we will also make the assumption that the images $\left\{Y_{i j}\right\}$ are independent for all $i$ and $j$. This first comes from the common assumption that the voice signals are independent, as they are produced by different physical instruments and acoustic processes. Second, independence across channels effectively boils down to discarding any phase dependencies. Even if it is arguable, we found in practice that discarding phase modeling in our case was both computationally efficient and did not harm the results.

Finally, we choose to model the signals using a Local Gaussian Model (LGM, [13, 14, 15]). In addition to assuming that the TF bins are independent, it assumes that each $Y_{i j}(\omega, t)$ is distributed with respect to a complex isotropic Gaussian distribution:

$$
Y_{i j}(\omega, t) \sim \mathcal{N}_{c}\left(0, \sigma_{i j}^{2}(\omega, t)\right)
$$

where the parameter $\sigma_{i j}^{2}(\omega, t)$ is called the Power Spectral Density (PSD) of $y_{i j}$. Finally, we assume that the PSDs of each voice are equivalent up to a scaling factor $\lambda_{i j}(\omega)$ :

$$
\sigma_{i j}^{2}(\omega, t)=\lambda_{i j}(\omega) P_{j}(\omega, t) \approx\left|Y_{i j}(\omega, t)\right|^{2},
$$

where $P_{j}(\omega, t) \geq 0$ is the latent PSD of voice $j$ shared across all channels $i$. The scalar $\lambda_{i j}(\omega)$ gives the amount of interference of voice $j$ into channel $i$ at frequency band $\omega$. For this reason, we define the frequency dependent interference matrix as $\Lambda(\omega)=\left[\lambda_{i j}(\omega)\right]_{i, j}$. Note that this model amounts to discarding phase dependencies between the different channels, while relating them through a common latent PSD for each voice.

\subsection{Separation method}

Because $X_{i}(\omega, t)$ is the sum of $J$ independent complex isotropic Gaussian random variables $Y_{i j}(\omega, t)$ as in (1), it is itself distributed as a complex isotropic Gaussian:

$$
X_{i}(\omega, t) \sim \mathcal{N}_{c}\left(0, \sum_{j=1}^{J} \lambda_{i j}(\omega) P_{j}(\omega, t)\right) .
$$

If the PSD $P_{j}$ is known for each $j$, and the frequency-dependent interference matrices $\Lambda(\omega)$ are known, it has been shown [13, $14,15]$ that the Minimum Mean-Squared Error (MMSE) estimate $\hat{Y}_{i j}(\omega, t)$ of any $Y_{i j}(\omega, t)$ is given by generalized Wiener filtering:

$$
\begin{aligned}
\hat{Y}_{i j}(\omega, t) & =\frac{\lambda_{i j}(\omega) P_{j}(\omega, t)}{\sum_{j^{\prime}=1}^{J} \lambda_{i j^{\prime}}(\omega) P_{j^{\prime}}(\omega, t)} X_{i}(\omega, t) \\
& \triangleq W_{i j}(\omega, t) X_{i}(\omega, t)
\end{aligned}
$$

where $\triangleq$ denotes a definition and $W_{i j}(\omega, t)$ is called the Wiener gain. However, for a given voice $j$, we are usually not interested in estimating $Y_{i j}$ for all recordings $i$. Rather, we want to obtain $Y_{i j}$ only for those microphone channels that were initially positioned to capture this voice. We define the channel selection function $\varphi(j) \subseteq$ $\{1, \ldots, I\}$ that indicates which images of voice $j$ we want to recover. This is to account for possibly complex scenarios where several microphones are assigned to a single voice signal, as in the case of a concert piano. In the following, the channel selection function $\varphi$ is assumed to be known and given by the user (typically a sound engineer) who knows the recording setup and can easily provide this information. Furthermore, we assume that $\varphi(j) \neq \emptyset$ for all $j=1, \ldots, J$, meaning that each voice is predominant in at least one microphone channel.

Thus, the objective of interference reduction is to estimate all $\left\{\hat{Y}_{i j}\right\}_{i \in \varphi(j)}$ for each voice $j$. The resulting waveforms are easily recovered through an inverse STFT. Note that by selecting the "most relevant" images for a specific voice, we also significantly reduce computation time.

\subsection{Parameter estimation algorithm}

In this section, we describe the KAMIR algorithm for Interference Reduction based on KAM [10, 11, 12]. As input, it takes the STFTs $X_{i}$ of the recorded signals and the channel selection function $\varphi$, as described in the preceding section. It returns estimates for the desired clean signals $\left\{\hat{y}_{i j}\right\}_{i \in \varphi(j)}$ for each voice $j$. To do this, it only needs to estimate the parameters for the Wiener filter (5): the PSDs $P_{j}$ of the voices and the interference matrices $\Lambda(\omega)$.

In a nutshell, KAMIR alternates between two distinct procedures in an iterative fashion. In a first separation step, the current parameters $\Lambda(\omega)$ and $P_{j}$ for each $j$ and $\omega$ are assumed known and fixed. Then, separation of the desired clean signals $\left\{\hat{Y}_{i j}\right\}_{i \in \varphi(j)}$ is performed for all voices $j$ through Wiener filtering (5). In a second parameter fitting stage, those separated signals are kept fixed and 


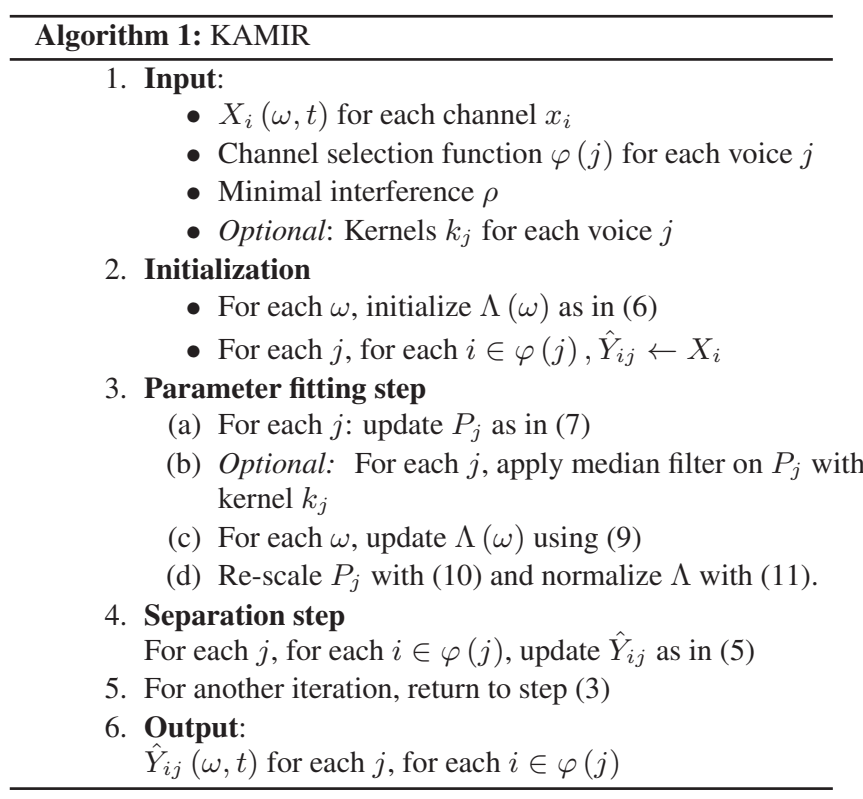

are assumed to be good estimates. The parameters $P_{j}$ and $\Lambda(\omega)$ are then re-estimated. Finally, the whole procedure is repeated until a stopping criterion is met, which is usually the imposed number of iterations. The KAMIR algorithm is summarized in Algorithm 1. We now discuss initialization and re-estimation of the parameters $P_{j}$ and $\Lambda(\omega)$.

Initialization (Algorithm Step 2). The elements of the interference matrices are initialized with:

$$
\forall(i, j, \omega), \lambda_{i j}(\omega)= \begin{cases}1 & \text { if } i \in \varphi(j) \\ \rho & \text { otherwise }\end{cases}
$$

where the parameter $\rho \in[0,1]$ is called the minimal interference, and corresponds to the minimum amount of interference we expect in any channel. The rationale for this initialization is the following: a voice $j$ which is associated to channel $i$ is given the maximum interference value 1 as it should ideally be fully captured in this channel; a voice $j$ which is not associated to channel $i$ is given the value $\rho$ as it should be only minimally captured in this channel. See Figure 2a for an example initialization of an interference matrix. Note that [3] can be understood as setting $\rho=1, \varphi(j):=\{j\}$ for $j \in\{1, \ldots, J\}$ with $I=J$, and performing only one iteration of KAMIR.

The estimate of the image $\hat{Y}_{i j}$ for each $j$ is initialized for $i \in$ $\varphi(j)$ with the observed STFT $X_{i}$ of channel $i$.

Power spectral density $P_{j}$ updates (Algorithm Steps 3a+3b). We know that the PSD of $y_{i j}$, is given by (3). Given the interference matrices $\Lambda(\omega)$ and image estimates $\hat{Y}_{i j}$, for a particular $i$ we can approximate $P_{j}(\omega, t) \approx \frac{1}{\lambda_{i j}(\omega)}\left|\hat{Y}_{i j}(\omega, t)\right|^{2}$. Because we may have multiple channels $i$ for which the previous expression is a good estimate, we take the average of this approximation across all channels in which voice $j$ is known to be predominant, yielding:

$$
P_{j}(\omega, t) \leftarrow \frac{1}{|\varphi(j)|} \sum_{i \in \varphi(j)} \frac{1}{\lambda_{i j}(\omega)}\left|\hat{Y}_{i j}(\omega, t)\right|^{2}
$$

where $|\varphi(j)|$ denotes the number of channels indicated by the selection function $\varphi$. (a)

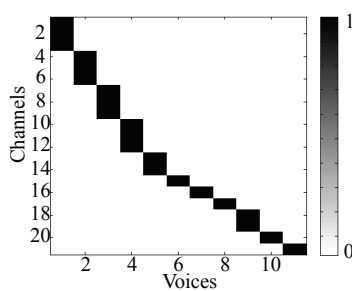

(b)

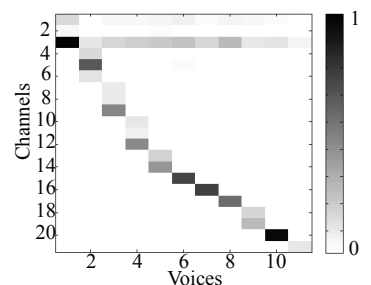

Fig. 2. Average interference matrix $\bar{\Lambda}=\frac{1}{N_{\omega}} \sum_{\omega} \Lambda(\omega)$ for an example with $I=21$ channels and $J=11$ voices (a) upon initialization, (b) after learning.

At this point, we can optionally apply a 2-dimensional median filter on $P_{j}$ as in KAM $[10,11,12]$ so as to enforce any knowledge we may know concerning a voice's local regularities. To do this, a user must provide a 2-D binary kernel $k_{j}$, with which $P_{j}$ is filtered. See the aforementioned references for examples of the choice of adequate kernels in audio.

Interference matrix $\Lambda(\omega)$ updates (Algorithm Step 3c).

According to our probabilistic model, the observed noisy channels $\left\{X_{i}(\omega, t)\right\}_{i, \omega, t}$ are independent and distributed according to (4). Given $P_{j}$, estimating each row $\Lambda_{i}(\omega)=\left[\lambda_{i 1}(\omega), \ldots, \lambda_{i J}(\omega)\right]$ of the matrix $\Lambda(\omega)$ can be done through the classical NMF methodology [7], as:

$$
\Lambda_{i}(\omega) \approx \underset{r_{1}, \ldots, r_{J}}{\operatorname{argmin}} \sum_{t=1}^{N_{t}} d_{\beta}\left(\left|X_{i}(\omega, t)\right|^{2}, \sum_{j=1}^{J} r_{j} P_{j}(\omega, t)\right),
$$

where $d_{\beta}$ stands for any appropriate cost-function such as the popular family of $\beta$-divergences, which notably includes KullbackLeibler $(\beta=1)$ and Itakura-Saito $(\beta=0)$. The main idea of using NMF here is to update $\Lambda(\omega)$ in a multiplicative fashion, so as to enforce nonnegativity. It can be shown that the corresponding update rule for each matrix element $\lambda_{i j}(\omega)$ is:

$$
\lambda_{i j}(\omega) \leftarrow \lambda_{i j}(\omega) \cdot \frac{\sum_{t=1}^{N_{t}} \hat{V}_{i}(\omega, t)^{\beta-2} V_{i}(\omega, t) P_{j}(\omega, t)}{\sum_{t=1}^{N_{t}} \hat{V}_{i}(\omega, t)^{\beta-1} P_{j}(\omega, t)},
$$

where $V_{i}(\omega, t) \triangleq\left|X_{i}(\omega, t)\right|^{2}$ and $\hat{V}_{i}(\omega, t) \triangleq \sum_{j} \lambda_{i j}(\omega) P_{j}(\omega, t)$. Note that $P_{j}$ is kept fixed during the NMF updates. After $\Lambda(\omega)$ has been updated, its entries are normalized ${ }^{1}$ to the interval $[\rho, 1]$. To achieve this, an easy procedure is to first re-scale $P_{j}(\omega, t)$ by:

$$
P_{j}(\omega, t) \leftarrow P_{j}(\omega, t) \sum_{i=1}^{I} \lambda_{i j}(\omega),
$$

and then to set:

$$
\lambda_{i j}(\omega) \leftarrow \max \left[\rho, \frac{\lambda_{i j}(\omega)}{\sum_{i^{\prime}=1}^{I} \lambda_{i^{\prime} j}(\omega)}\right] .
$$

An example of a learned interference matrix is shown in Figure $2 b$.

\footnotetext{
${ }^{1}$ All results reported in Section 3 use the normalization as in Equations (10) and (11). However, we later found that normalizing such that the total weight for each channel equals one (by skipping Equation (10) and changing the denominator in Equation (11) to $\sum_{j^{\prime}=1}^{J} \lambda_{i j^{\prime}}(\omega)$ ), leads to more reasonable results in $\Lambda$. We suggest that this alternate normalization be used when implementing the algorithm.
} 

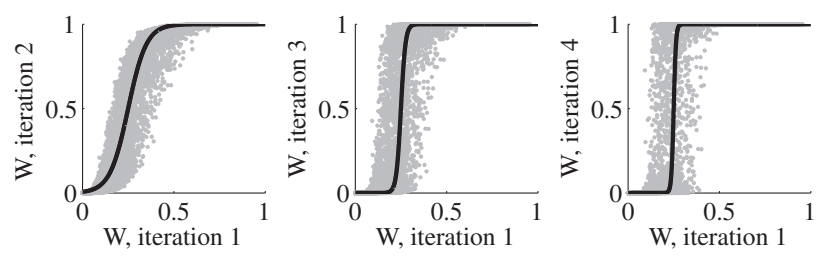

Fig. 3. Evolution of the gains of the Wiener filters for iteration 2, 3 and 4 with respect to iteration 1 .

\subsection{Computationally Effective Approximation}

If no kernels $k_{j}$ are provided to filter the PSD estimates $P_{j}$ at step $3 \mathrm{~b}$ of KAMIR, we observe that the Wiener gains $W_{i j}$ in (5) evolve in a very particular fashion during the iterations of KAMIR. To illustrate this, we display the Wiener gains at iterations 2-4 as a function of their value at iteration 1 as scatter plots in Figure 3. We see that there is a clear tendency towards binarization of the Wiener gains with increasing iterations. This can effectively be modeled as applying a sigmoidal function $g$ to the initial Wiener masks $W_{i j}$, as demonstrated by the bold curves in Figure 3. The sigmoidal gain function $g$ is given by:

$$
g(x)=1-\frac{1}{1+\exp (s \cdot(x-\tau))},
$$

where $s$ and $\tau$ are slope and a threshold parameters, respectively. The slope of the sigmoid function approximates the number of iterations, and $\tau$ is related to the minimal interference $\rho$.

Hence, to simulate applying several iterations of KAMIR, instead of actually making the computations, we can simply replace $W_{i j}(\omega, t)$ in (5) by $g\left(W_{i j}(\omega, t)\right)$. In this way, only one iteration is needed to update the inference matrices $\Lambda(\omega)$ and proceed to interference reduction. Note that this approximation is only valid if no kernels $k_{j}$ are provided for median filtering the PSD estimates. This is because median filtering induces dependencies between different TF bins, causing the sigmoidal approximation to become a poor model.

\section{EVALUATION}

We compared KAMIR's performance for five different parameter settings with the algorithm from [4] on an orchestra recording featuring 21 microphones with up to 11 voices ${ }^{2,3}$. Throughout the experiments, we used $\beta=0$ and omitted the kernel median filtering (Algorithm Step 3b). Because the recording session used a live setup featuring interference in all microphones, the standard evaluation metrics for blind source separation $[17,18]$ were not applicable, as they require a clean reference signal. Instead, we evaluated the performance of each setting in a perceptual study involving 21 listeners using five different microphone signals (items). Listeners gave two ratings for each item and setting: first, a rating of "how well the reduction of interference was accomplished" (Figure 4a), and second, a rating for the overall quality, considering the success of both the interference reduction and the sound quality (Figure $4 \mathrm{~b}$ ).

As can be seen in Figure 4b, the Kokkinis 2012 algorithm [4] has the tendency to perform slightly better in terms of overall sound quality, especially for items \#1 and \#4. However, we also see that

\footnotetext{
${ }^{2}$ We want to thank Elias Kokkinis who has provided the code for his algorithm and a parameter setting specifically tuned for this recording.

${ }^{3}$ The recording is an excerpt of the opera "Der Freischütz" recorded by the project Freischütz Digital (www. freischuetz-digital.de) [16].
}

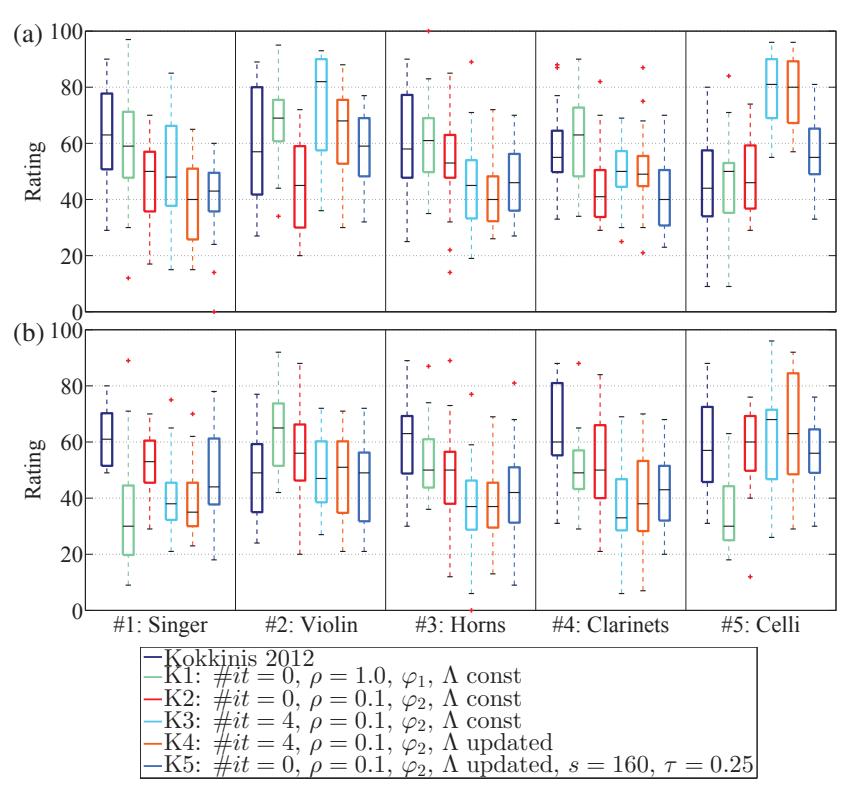

Fig. 4. Listening test results. The boxes show the interquartile range, black bars the median, and red crosses outliers. The channel selection function $\varphi_{1}(j)=\{j\}$, whereas $\varphi_{2}$ is initialized according to the microphone setup and groups channels belonging to the same voices. (a) Interference Ratings. (b) Overall quality ratings.

the different versions of KAMIR perform favorably as well, especially when the model benefits from several microphones for one voice signal. For example, for item \#5, the three microphones used for the cello section lead to improvements in the interference reduction without a reduction in the overall quality. See the parameter settings $K 3$ and $K 4$ compared to the approaches in Figure $4 \mathrm{a}$ and b.

Another interesting observation is that applying several iterations and learning the interference matrices $\Lambda(\omega)$, improves the interference reduction of KAMIR in certain scenarios (see items $\# 2, \# 4, \# 5$ with the parameter settings $K 3$, and $K 4$ compared with $K 2$ in Figure $4 a$ ). However, items $\# 1, \# 3$, \#4 in Figure $4 b$ indicate an inverse tendency for the overall quality ratings of $K 2$, $K 3$ and $K 4$. This is not surprising, because by suppressing more interferences, artifacts or distortions are more likely to be introduced into the signals. Finally, we want to note that the computationally effective approximation K5 performs similarly to the other parameter settings. The sound material of the listening test and an implementation of the algorithm can be found at $[19,20]$.

\section{CONCLUSIONS}

In this paper, we have proposed a simple, yet effective algorithm called KAMIR for interference reduction in multi-channel live recordings. It is based on iteratively estimating the spectrograms of the desired clean voices and the interference in the microphone channels. Our evaluation indicates that in addition to being very simple to implement, KAMIR produces separated voices that have good perceptual quality. It furthermore provides a practical way to trade-off interference reduction and distortion, notably through the number of iterations. Finally, we have shown that in some cases, the impact of several iterations of KAMIR can be predicted from the result of its first iteration, yielding a computationally effective technique for interference reduction. Future work includes evaluation of the use of kernels within the KAMIR algorithm. 


\section{REFERENCES}

[1] Alice Clifford and Joshua Reiss, "Microphone interference reduction in live sound," in Proceedings of the International Conference on Digital Audio Effects (DAFx), 2011.

[2] Christian Uhle and Josh Reiss, "Determined source separation for microphone recordings using IIR filters," in Audio Engineering Society Convention, Audio Engineering Society (AES), Ed., November 2010.

[3] Elias K. Kokkinis and John Mourjopoulos, "Unmixing acoustic sources in real reverberant environments for closemicrophone applications," Journal of the Audio Engineering Society, vol. 58, no. 11, pp. 907-922, 2010.

[4] Elias K. Kokkinis, Joshua D. Reiss, and John Mourjopoulos, "A Wiener filter approach to microphone leakage reduction in close-microphone applications," IEEE Transactions on Audio, Speech \& Language Processing, vol. 20, no. 3, pp. 767-779, 2012.

[5] Elias Kokkinis, Alexandros Tsilfidis, Thanos Kostis, and Kostas Karamitas, "A new DSP tool for drum leakage suppression," in Audio Engineering Society Convention, Audio Engineering Society (AES), Ed., 2013.

[6] Julio J. Carabias-Orti, Maximo Cobos, Pedro Vera-Candeas, and Francisco J. Rodriguez-Serrano, "Nonnegative signal factorization with learnt instrument models for sound source separation in close-microphone recordings," EURASIP Journal on Advances in Signal Processing, vol. 2013, pp. 184, 2013.

[7] Andrzej Cichocki, Rafal Zdunek, Anh Huy Phan, and Shunichi Amari, Nonnegative Matrix and Tensor Factorizations: Applications to Exploratory Multi-way Data Analysis and Blind Source Separation, Wiley Publishing, Sept. 2009.

[8] Emmanuel Vincent, Nancy Bertin, Rémi Gribonval, and Frédéric Bimbot, "From blind to guided audio source separation: How models and side information can improve the separation of sound," IEEE Signal Processing Magazine, vol. 31, no. 3, pp. 107-115, May 2014.

[9] Antoine Liutkus, J-L. Durrieu, Laurent Daudet, and Gaël Richard, "An overview of informed audio source separation," in Workshop on Image Analysis for Multimedia Interactive Services WIAMIS, Paris, France, July 2013, pp. 1-4.

[10] Antoine Liutkus, Derry Fitzgerald, Zafar Rafii, Bryan Pardo, and Laurent Daudet, "Kernel Additive Models for Source Separation," IEEE Transactions on Signal Processing, June 2014.

[11] Antoine Liutkus, Zafar Rafii, Bryan Pardo, Derry Fitzgerald, and Laurent Daudet, "Kernel spectrogram models for source separation," in Hands-free Speech Communication and Microphone Arrays (HSCMA), Nancy, France, May 2014.

[12] Derry Fitzgerald, Antoine Liutkus, Zafar Rafii, Bryan Pardo, and Laurent Daudet, "Harmonic/percussive separation using Kernel Additive Modelling," in Irish Signals \& Systems Conference (IET), 2014.

[13] Antoine Liutkus, Roland Badeau, and Gäel Richard, "Gaussian processes for underdetermined source separation," IEEE Transactions on Signal Processing, vol. 59, no. 7, pp. 3155 3167, July 2011.

[14] Laurent Benaroya, Frédéric Bimbot, and Rémi Gribonval, "Audio source separation with a single sensor," IEEE Transactions on Audio, Speech and Language Processing, vol. 14, no. 1, pp. 191-199, Jan. 2006.
[15] Ngoc Q. K. Duong, Emmanuel Vincent, and Rémi Gribonval, "Under-determined reverberant audio source separation using a full-rank spatial covariance model," IEEE Transactions on Audio, Speech, and Language Processing, vol. 18, no. 7, pp. 1830 -1840, Sept. 2010.

[16] Meinard Müller, Thomas Prätzlich, Benjamin Bohl, and Joachim Veit, "Freischütz Digital: a multimodal scenario for informed music processing," in Proceedings of the 14th International Workshop on Image and Audio Analysis for Multimedia Interactive Services (WIAMIS), Paris, France, 2013, pp. $1-4$.

[17] Emmanuel Vincent, Rémi Gribonval, and Cédric Févotte, "Performance measurement in blind audio source separation," IEEE Transactions on Audio, Speech, and Language Processing, vol. 14, no. 4, pp. 1462-1469, 2006.

[18] Valentin Emiya, Emmanuel Vincent, Niklas Harlander, and Volker Hohmann, "Subjective and objective quality assessment of audio source separation," IEEE Transactions on Audio, Speech, and Language Processing, vol. 19, no. 7, pp. 20462057, 2011.

[19] Thomas Prätzlich, Rachel Bittner, Antoine Liutkus, and Meinard Müller, "Accompanying website: Kernel additive modeling for interference reduction in multi-channel music recordings," http: //www. audiolabs-erlangen.de/ resources/MIR/2015-ICASSP-KAMIR, 2014.

[20] Antoine Liutkus, “Implementation of KAMIR," http:// www.loria.fr/ aliutkus/kamir/, 2014. 